\title{
IMPLEMENTED TO BLUETOOTH CONNECTIONS - FIXED SITUATION
}

\author{
Nirmla Sharma \\ Department of Computer Science, King Khalid University, Saudi Arabia
}

\begin{abstract}
Bluetooth has basically complete requirement for short collection wireless transportations consuming unrestricted manufacturing, technical, medicinal (ISM) $2.4 \mathrm{GHz}$ wireless group. Bluetooth works on seventy-nine frequencies in $2.4 \mathrm{GHz}$ group by $1 \mathrm{MHz}$ transporter arrangement. Individually scheme completes occurrence skipping with 1600 hops/s in Pseudo Random method. Bluetooth relates Frequency Hopping Systems (FHSS) used for intervention modification Frequency Hopping Code Division Multiple Access (FH-CDMA for departure of systems). Additional feature is described in this paper around Bluetooth's radio layer. An actual significant term in setting of Bluetooth has Piconet. A Piconet has gathering of Bluetooth strategies which were corresponding to similar skipping arrangement. Bluetooth connections are implemented by different techniques. These techniques are using to wireless networks.
\end{abstract}

Keywords:

Bluetooth, Connection, Piconet, techniques and Wireless networks

\section{INTRODUCTION}

Bluetooth technology has basically wire standby scheme it offers complete connection toward standing statistics systems combine with ad hoc construction device used for selection of strategies now numerous formations. Bluetooth conditions have been planned through accepting that eliminating restriction of wire and executing radio association has individual helpful uncertainty wireless technology has financial and strong wire it exchanges, or radio association proposals extra benefits. It has presented Bluetooth protocols and challenging actions.

\subsection{BLUETOOTH USE SPECIAL INTEREST GROUP (SIG)}

Bluetooth Special Interest Group (SIG) has contained of selected work out, broadcastings and system activities. It has quantified Bluetooth's procedures using request summaries. Collection has dynamic improvement of expertise with transporting in market place. Bluetooth SIG contains organizer businesses companies Com, Ericsson, IBM, Intel, Lucent, Microsoft, Motorola, Nokia, and Toshiba, along with over 2000 Adopter and Subordinate members.

It wants this authorization to achieve slightly Bluetooth statement like inviting a connection, accepting a connection, and relocating information. Flexibility between individuals has continuously developed and wireless technologies for speech and statistics have changed quickly throughout the historical ages. Numerous electronic devices for household, individual and commercial practice have been offered to the marketplace during current years but no extensive skill has addressed the requirements of linking individual devices in Personal Area Networks (PAN). The request for an organization that could simply assign devices for assignment of information and speech finished little reserves - without cables - produced stronger [1].

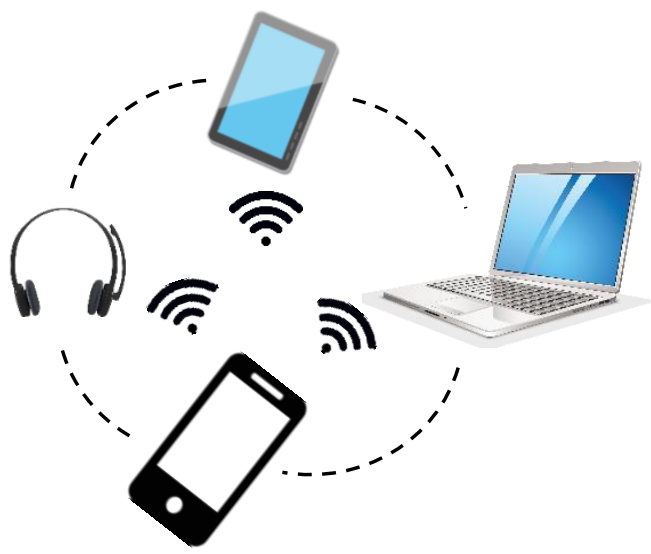

Fig.1. Bluetooth topology performance makes Piconet

Raw statistics frequency has $1 \mathrm{Mbit} / \mathrm{s}$ through speech stations associate $64 \mathrm{kbit} / \mathrm{s}$ and unequal connection of $721 \mathrm{kbit} / \mathrm{s}$ route, though allowing $57.6 \mathrm{kbit} / \mathrm{s}$ arrival directions or $432.6 \mathrm{kbit} / \mathrm{s}$ symmetric associations. Unique Bluetooth scheme has performance as per master, directing entirely circulation in Piconet, although entirely extra strategies performance as per slaves. Master has distinct scheme that recruit's association process toward creates Piconet. Distinct unique master happens individually Piconet. Least Piconet contains of objective dual strategies (point-to-point) - single master and unique slave. Dynamic 7-slaves could remain forceful in Piconet. Strategies could be retained in Common method and master may be backing up to 255 like strategies (point-to-multipoint). Different scheme could be practice User Service Discovery (USD) to control what system facilities are offered from numerous associated strategies [2][3].

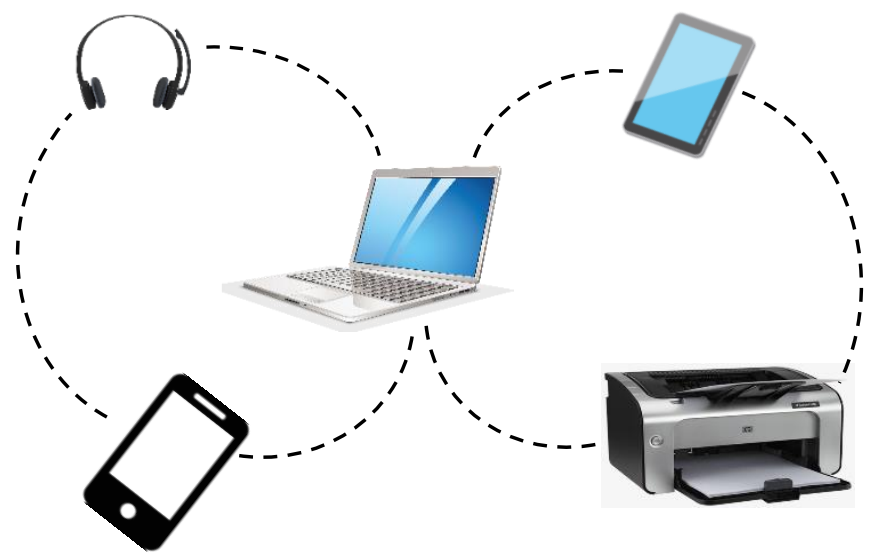

Fig.2. Bluetooth Scatternet involves collection of Piconets overlying analysis 
The Fig.2 is represented to implement the frequency transfer of different devices. A collection of piconets through overlapping parts of attention is called scatternet. Every piconet is recognized through dissimilar occurrence skipping order. Bluetooth scheme might contribute in dissimilar Piconets delivered it has individual dynamic Piconet using this period. Scheme could performance as per slave in dissimilar Piconets, however as per master in individual distinct Piconet [4] [5].

\subsection{BLUETOOTH TECHNOLOGY}

The advantage and control of Bluetooth technology, four practice representations are below:

Ultimate Headset: The headset permits you to practice your mobile handset smooth uncertainty the handset is in a folder.

Automatic Synchronization: Automatic synchronization of almanacs, address books, e-mails, etc. is a long-awaited article. As soon as you are arrived in workplace, almanac on your handset will be routinely reorganized toward remain reliable by unique in your desktop PC, or vice versa.

Internet Bridge: Someplace you are, Bluetooth wireless technology contracts you surf the Internet without any cable connections. It may contact the net wirelessly consuming moreover a movable computer or a cellular handset.

Three-in-One Phone: At home your handset may purpose by way of cordless handset (fixed-line charge). Once they are on the transfer, your handset may purpose by way of a cellular handset (cellular charge) [6].

\subsection{MOTIVATION CONNECTION}

\section{AND BLUETOOTH}

\subsubsection{Bluetooth Wireless and Association Switch:}

Wireless skipping arrangement has recycled toward conflict intervention and disappearing. Piconet master defines skipping arrangement and technique has defined through master element's scheme timer. As part from association procedure, slave would accept technique statistics after master, containing its modern timer and skipping arrangement. Motivational process confirms entirely Bluetooth strategies in Piconet and synchronized.

Extra methods have procedure of host processor and firmware to tool baseband, trust wireless distinct. Regardless of hardware execution, Bluetooth practice stack order statistics are preserved [7].

\subsubsection{Connection Controller:}

Administrator has responsible used for guilty particulars connect and when, whatever details delay to force rate of interconnect and switch control process. Baseband resolves comprise Piconet scheme change drives, approaches of process, and intermediate contact purposes. Switch purposes contain association design, occurrence skipping arrangement collection and method. Control change and protected procedure are occurrences of procedure styles. Standard contact resolves hold purposes like asking, package brands, package treating, and connection brands like Asynchronous Connectionless and Synchronous Connection Oriented (ACL and SCO).

Link Management Protocol - Protocol Data Unit (LMP_PDU) has connection use directing in business with link manager in additional scheme, association administrator would react with following LMP_PDU operation order. Consecutively, unloading association administrator replies through LMP accepted or LMP not accepted PDU. If LMP not accepted PDU has directed, purpose used for not tolerant business has delivered [8].

\subsubsection{Bluetooth Connections are Recycled:}

- Reduces cables and wires among equally stationary and movable devices [1]

- Enables equally information and speech statement

- Proposals the option of ad hoc systems and transports informal synchronicity among altogether individual electronic devices.

Bluetooth wireless technology stops this vital statement essential by its capacity to connect both speech and information wirelessly, consuming a normal low-power, low-cost technology which may be combined in entirely devices and therefore permit entire flexibility [3].

\subsection{PROBLEM STATEMENT}

Bluetooth module has executes of hardware, wireless and association controller while firmware connection manager. Wireless and connection controller beside by baseband purposes were primary shares of Bluetooth requirement near established. At current, wireless has usually distinct chip after Connection Controller and Connection Manager, while objective of record producers has delivered distinct bit, short price resolution, universe and control aware projects. Connection controller is hardware administrator for wireless and host of Baseband procedure drives.

\section{BLUETOOTH TELEPHONY CONTROL PROTOCOL SPECIFICATION (TCS)}

Bluetooth Telephony Control protocol Specification (TCS) describes in what way handset demands would be directed through Bluetooth relation. It springs rules for indicating desirable to usual active composed point-to-point, and point-tomultipoint requests. It too offers way to direct DTMF qualities through Bluetooth association. TCS is modified after ITU. Abbreviation recycled used for telephony switch does not acceptable in through agreement recycled in recreation of Bluetooth mass. Through subsequent rest of mass identification agreements, this would be named TCP; though it was certain that it would conflict with Internet Transport Control Protocol (TCP).

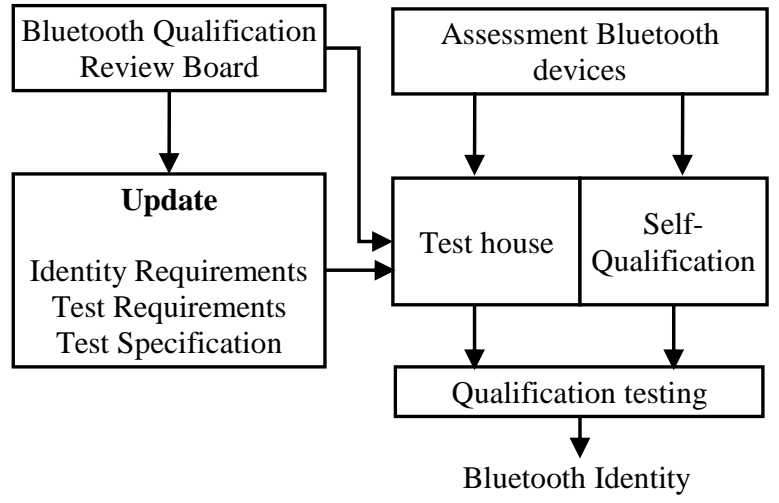

Fig.3. Complete requirement procedure confirms that Bluetooth scheme observe with Bluetooth requirement 
Requirement procedure confirms that Bluetooth scheme submit with Bluetooth Requirement, thus allowing interoperability among strategies. Bluetooth requirement is essential condition of logical assets certificate for Bluetooth radio technology. Requirement has too essential to relate Bluetooth symbol to creation (subject to distinct symbol contract). Requirement is not sort support producers of Bluetooth scheme might too drive complete usual actions of controlling style support in individually county or section where it desires to retail creation [9].

\begin{tabular}{|c|c|c|c|}
\hline \multicolumn{4}{|c|}{ Bluetooth SIG/Regulatory Group/TF2 } \\
\hline \multicolumn{4}{|c|}{$\nabla$} \\
\hline \multicolumn{4}{|c|}{ Bluetooth Qualification Review Board (BQRB) } \\
\hline$\downarrow$ & $\downarrow$ & $\downarrow$ & $\downarrow$ \\
\hline $\begin{array}{c}\text { Bluetooth } \\
\text { Qualification } \\
\text { Administrator } \\
\text { (BQA) }\end{array}$ & $\begin{array}{c}\text { Bluetooth } \\
\text { Qualification } \\
\text { Test Facility } \\
\text { (BQTF) }\end{array}$ & $\begin{array}{c}\text { Bluetooth } \\
\text { Qualification } \\
\text { Body (BQB) }\end{array}$ & $\begin{array}{c}\text { Bluetooth } \\
\text { Technical } \\
\text { Advisory Board } \\
\text { (BTAB) }\end{array}$ \\
\hline
\end{tabular}

Fig.4. Collection of Bluetooth requirement figures supervises correct assessment and authentication of Bluetooth scheme

Numerous helpful requirement forms direct Bluetooth assessment and confirmation shows in Fig.4:

- Bluetooth Qualification Review Board (BQRB) - it involves of representatives since individually Bluetooth supporter whose core assignment is creating strategy.

- Bluetooth Qualification Administrator (BQA) - It liable for directing Bluetooth Requirement Database with BQRB.

- Bluetooth Qualification Test Facility (BQTF) - some assessment ability credited by BQRB to assessment Bluetooth schemes.

- Bluetooth Qualification Body (BOB) - individual certified by $\mathrm{BQRB}$ to deliver facilities to adopter pursuing Bluetooth scheme requirement. BOB is liable for testing statements and papers beside desires, revising creation assessment reports, and catalog scheme to certified record of Bluetooth practiced scheme

- Bluetooth Technical Advisory Board (BTAB) - opportunity containing of altogether BQBs and BQTFs, liable for directing BQRB scheduled practical substances connecting to assessment desires, experiment circumstances, assessment qualifications and assessment tools [10].

Bluetooth Qualification Review Board needs recognized 4assessment groups, A to D:

- Group ' $A$ ' - Bluetooth scheme could individual be verified, authenticated and commercially presented qualification test tools by BQTF.

- Group 'B and C'- Associate or Bluetooth Qualification Test Ability could assessment Bluetooth scheme consuming ordinary assessment tools.

- Category ' $\boldsymbol{D}$ ' - is introductory assessment instance by no certified requirement assessment. Determination of station is to update some producer near future assessment situation. Until certified procedure conformance assessment schemes developed offered, Blue Elements are recycled to create assurance in lesser level procedures.

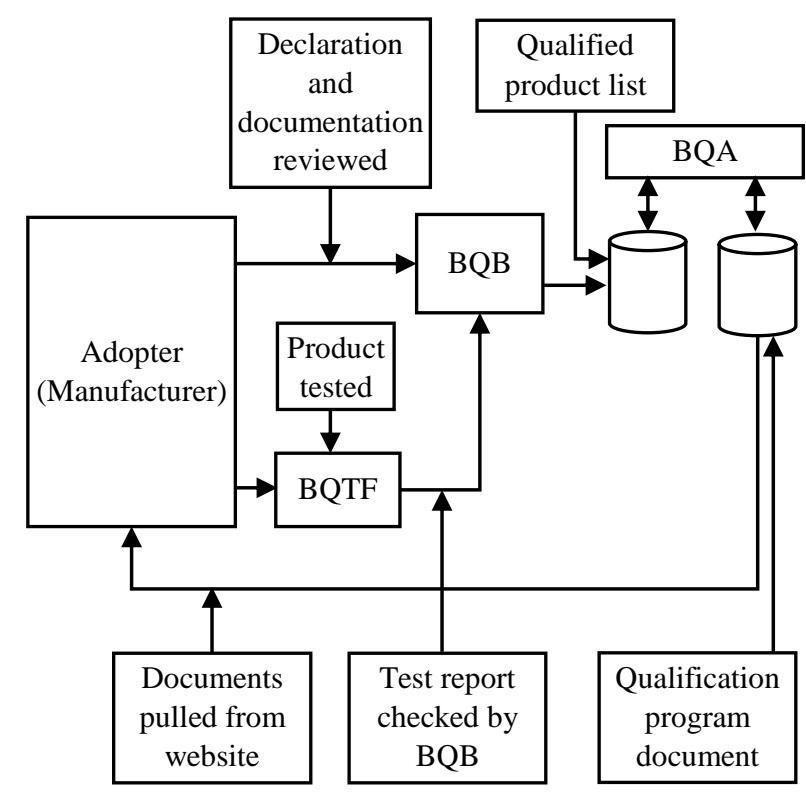

Fig.5. Required Bluetooth challenging includes an inflexible set of challenging, certification, and restriction jobs

The Fig.5 displays technical movement complicated with succeeding Bluetooth scheme. Bluetooth protocol sample and tools, like range analyzers, are compound tools naturally recycled by Bluetooth Qualification Test Services. Though, Bluetooth procedure analyzers are direction tenth of price of Bluetooth procedure sample. Procedure analyzer is frequently recycled for scheme development, accurate and prequalification examination. Specific ability of Bluetooth procedure analyzer is accurate of hardware/software accumulation anywhere assured tools exactly produce prearranged faults for assessment and correct of developed level protocols [11].

\section{BENEFITS AND COMPARED TO THE EXISTING SYSTEM}

Bluetooth is a wireless connection technology that permits you to connect numerous devices in your household. As Wi-Fi, it may practice Bluetooth to exchange records and run electronics. Wireless receivers, cell phones and smooth gaming supports may altogether practice Bluetooth in unique method or extra.

Simple Setup: Situation active a Bluetooth connection among two devices is rapid and informal. The careful line for responsibility thus differs depending on your strategies, then to link two devices you essential create one discoverable though the extra one images.

Compatibility: A Bluetooth receiver is well-matched with any extra device that wires Bluetooth, regardless of create, perfect or strategy. It may practice it with your movable handset or couple it with your gaming console or PC for informal conversation connected.

Less Hardware: Visibly the lack of cables is a advantage of Bluetooth, then it's smooth fewer huge than Wi-Fi. To make a wireless system in household consuming Wi-Fi will essential a router to established the contact opinion. Entirely statement among dual devices on a wireless system might drive over wireless router. 
Security: Bluetooth has characteristic security in dual methods. The primary is that it is not continuously broadcasting, dissimilar a Wi-Fi association. Once it requests to associate dual devices via Bluetooth it established them to be observable, too occasionally called "discoverable". This visibility is individual essential till the devices have been combined. Once combined, it may try off visibility and retain the device secure to original connections. The second level of security is that in record cases it will essential to allow the connection consuming a PIN or code to connect dual devices [12].

The Bluetooth wireless technology comprises hardware, software and interoperability desires. It has been accepted not only by all main companies in the telecom, computer and home entertainment industry, but also in such different parts as the automotive commerce and fitness care, automation and figures, etc. - virtually all parts of the reduced. It is probable that earlier lengthy Bluetooth technology will be constructed into hundreds of millions of electronic devices all over the creation [13].

\section{CONCLUSION AND FUTURE WORK}

Bluetooth protocol sample and tools, like range analyzers, are compound tools naturally recycled by Bluetooth Qualification Test Services. Though, Bluetooth procedure analyzers are direction tenth of price of Bluetooth procedure sample. Specific ability of Bluetooth procedure analyzer is accurate of hardware/software accumulation anywhere assured tools exactly produce prearranged faults for assessment and correct of developed level protocols. A Piconet has gathering of Bluetooth strategies which were corresponding to similar skipping arrangement. All figures show group of policies with dissimilar characters. One method in Piconet could performance master $(M)$, all additional policies associated to master need performance slaves (S). Master controls skipping design in Piconet and slaves must to coordinate to this design. Bluetooth contacts to physical position and switch registers. It could be recognized hardware and software limit.

The impression remained that a small radio constructed into mutually the cellular handset and the laptop would switch the unwieldy wire recycled currently to connect the dual devices. A time future the engineering effort started and the correct prospective of the technology started to form. Outside unchecking devices by exchanging wires, the radio technology presented options to develop a common connection to current statistics systems, a peripheral interface, and a device to method minor isolated ad hoc collections of associated devices missing from immovable network infrastructures. This technology is known currently equally Bluetooth technology.

\section{REFERENCES}

[1] Nokia and Bluetooth: What is Bluetooth?, Available at: https://www.nokia.com/en_us/phones/support/nokia-6user-guide/bluetooth.

[2] Bluetooth, "Core Specifications", Available at: https://www.bluetooth.com/specifications/bluetooth-corespecification.

[3] Bluetooth Special Interest Group, "Bluetooth technology to gain longer range faster speed mesh networking in 2016," November 112015.

[4] Bluetooth Special Interest Group, Available at: https://www.bluetooth.com/

[5] J Bray and C.F. Sturman, "Bluetooth-Connect Without Cables", Prentice-Hall, 2001.

[6] K. H. Chang, "Bluetooth: A Viable Solution for IOT? [Industry Perspectives]", IEEE Wireless Communications, Vol. 21, No. 6, pp. 6-7, 2014.

[7] J. Nieminen, et.al, "Networking Solutions for Connecting Bluetooth Low Energy Enabled Machines to the Internet of Things", IEEE Network, Vol. 28, No. 6, pp. 83-90, 2014.

[8] C. J. Mathias, "Bluetooth is Dead", Available at: https://www.eetimes.com/document.asp?doc_id=1147339

[9] T. Mueller, "Bluetooth Security Architecture White Paper", Available

at: http://www.afn.org/ afn48922/downs/wireless/1c11600.pdf

[10] K. Feher, "Wireless Digital Communications- Modulation and Spread Spectrum Applications", Prentice-Hall, 1995. 